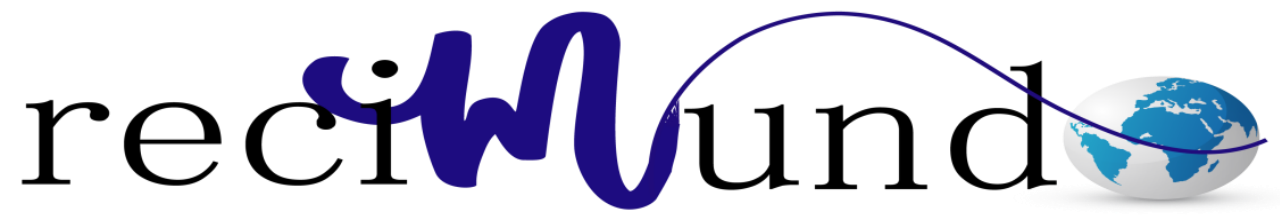

Revista Científica Mundo de la Investigación y el Conocimiento

Eva María de Lourdes Loaiza Massuh ${ }^{\text {a; }}$ Holger Renato Ponce Arreaga b; Hypathia Verónica Fiallos Veintimilla ${ }^{\mathrm{c}}$

Análisis de Emprendimiento de Yogurt a base de Arazá en la Ciudad de Guayaquil

Revista Científica Mundo de la Investigación y el Conocimiento. Vol. 2 núm., especial, mayo, ISSN: 2588-073X, 2018, pp. 847-876

DOI: 10.26820/recimundo/2.esp.2018.847-876

Editorial Saberes del Conocimiento

Recibido: 05/12/2017 Aceptado: $15 / 02 / 2018$

a. Universidad de Guayaquil-Docente; eva.1oaizam@ug.edu.ec

b. Universidad de Guayaquil- Analista de Planificación y Proyectos de Inversión; holger.poncea@ug.edu.ec

c. Universidad de Guayaquil-Docente; hypathia.fiallosv@ug.edu.ec 


\section{Análisis de Emprendimiento de Yogurt a base de Arazá en la Ciudad de Guayaquil}

Vol. 2, núm. Esp., (2018)

Eva María de Lourdes Loaiza Massuh; Holger Renato Ponce Arreaga; Hypathia Verónica Fiallos Veintimilla

\section{Introducción.}

El presente artículo es un aporte práctico sobre la elaboración de un nuevo yogurt dietético a base de Arazá, que es una fruta amazónica. Partiendo del conocimiento de que el Arazá posee nutrientes y vitaminas que son necesarias en la dieta alimenticia de las personas, además ayuda a combatir múltiples enfermedades debido a que es considerada una fuente de energía y salud que el cuerpo necesita.

El yogurt también posee muchos beneficios para mejorar la salud, uno de los principales es su poder de restablecer la flora intestinal, y así contribuir a una vida saludable. A través de estos beneficios que posee tanto el Arazá como el yogurt, ha surgido la idea de elaborar este innovador proyecto, el cual permitirá satisfacer las necesidades del consumidor, otro factor que permite la introducción del producto en el mercado es la inexistencia de un yogurt dietético a base de Arazá.

El desarrollo del presente proyecto está dirigido a los habitantes de la ciudad de Guayaquil. El propósito del mismo es ofrecer al público un yogurt dietético con la finalidad de cubrir las necesidades de los consumidores que padecen diversas enfermedades debido a un desorden alimenticio y a la rutina diaria.

\section{PLANTEAMIENTO PROBLEMA}

Hoy en día estamos inmersos en un mundo en el que llevamos un ritmo de vida agitado, sea por trabajo, por estudios, o por cualquier otra actividad que realicemos en nuestra vida cotidiana o muy al contrario llevamos una vida sedentaria; dejamos a un lado el cuidado de nuestra salud 


\section{Análisis de Emprendimiento de Yogurt a base de Arazá en la Ciudad de \\ Guayaquil}

Vol. 2, núm. Esp., (2018)

Eva María de Lourdes Loaiza Massuh; Holger Renato Ponce Arreaga; Hypathia Verónica Fiallos

Veintimilla

debido a que tenemos una alimentación poco nutritiva, por ingerir comida chatarra alta en grasa, carbohidratos y azucares, lo cual conlleva a altas tasas de obesidad y sobrepeso que en la actualidad se presentan desde temprana edad.

Según la OMS (Organización Mundial de la Salud) define "Sobrepeso" y "Obesidad "como una acumulación anormal o excesiva de grasa que puede ser perjudicial para la salud. La mala ingesta de alimentos conlleva grandes consecuencias que a futuro podemos lamentar en nuestra salud, puesto que una persona obesa o con sobrepeso llega a presentar problemas de estreñimiento, del corazón, hipertensión, diabetes, problemas en el sistema óseo e incluso cáncer de hígado, vesícula, riñones y colon.

Todas estas enfermedades se pueden prevenir haciendo conciencia del ritmo de vida que estamos llevando en cuanto a la mala alimentación y falta de ejercicio, bajando el consumo de calorías y aumentando la ingesta de frutas, cereales, legumbres y verduras.

En el mercado actual los productos elaborados a base de arazá no han sido explotados con mayor dinamismo para el consumo humano, por lo cual el uso e implementación de esta fruta es escasa en la alimentación diaria de niños, jóvenes y adultos.

\section{JUSTIFICACIÓN}

El cuidado de la salud depende únicamente de cada uno de nosotros y dando importancia a lo antes mencionado, se han investigado las valiosas propiedades de una fruta no tan común llamada Arazá, la cual posee entre 90\% a 94\% de agua como principal componente, además de 


\section{Análisis de Emprendimiento de Yogurt a base de Arazá en la Ciudad de Guayaquil}

Vol. 2, núm. Esp., (2018)

Eva María de Lourdes Loaiza Massuh; Holger Renato Ponce Arreaga; Hypathia Verónica Fiallos Veintimilla

contener calcio, magnesio, fosforo y altos niveles de vitamina $\mathrm{C}$ que ayudan a aumentar las defensas.

El fruto de arazá, de la región amazónica colombiana, se proyecta día tras día como un elemento productivo tendiente a mejorar el renglón socioeconómico de la región; sus características organolépticas lo hacen apetecible y sus componentes nutricionales prometen un beneficio para la salud de las personas principalmente de esta región que lo consumen dentro de su dieta regular. La pulpa del fruto se caracteriza por poseer $\mathrm{pH}(2,0),{ }^{\circ} \mathrm{Brix}(4,0)$ bajos y unos contenidos en base seca de carbohidratos entre 70 a 80,6\%, proteína6,0 a 10,9\%, pectina 3,4\%, vitamina $\mathrm{C} 0,77$ a 7,4\%, calcio 0,16 a 0,22\%, magnesio de 0,08 a 0,12\%, entre otros. (Ariza, 2001)

Esta fruta es ideal para preparar batidos, mermeladas, postres, helado; pero esta vez se tomará un único enfoque: la creación de yogurt a base de arazá. ¿Por qué creamos yogurt de arazá? porque el yogurt en conjunto con la fruta arazá crea una fuente de proteínas mediante la asimilación de nutrientes, además de que por sus amplias propiedades estabiliza la flora intestinal, por su sabor cítricos favorece a la reducción de grasas lo cual resulta un buen aliado en contra del sobrepeso y la obesidad, ayuda a mejorar el aspecto de la piel debido a su gran cantidad de agua, combate la diarrea y el estreñimiento.

En la Amazonia existe una enorme variedad de especies frutícolas nativas de gran potencial que, si se explotan racionalmente, podrían contribuir al desarrollo local. Entre las frutícolas regionales, el arazá despierta cierto interés por las cualidades organolépticas del fruto y por el índice de producción de la planta. Su fruto suculento posee un aroma y sabor agradable, 


\section{Análisis de Emprendimiento de Yogurt a base de Arazá en la Ciudad de \\ Guayaquil}

Vol. 2, núm. Esp., (2018)

Eva María de Lourdes Loaiza Massuh; Holger Renato Ponce Arreaga; Hypathia Verónica Fiallos

Veintimilla

pudiendo ser consumido en forma de refresco, dulce, néctar, jalea, licor, yogurt, etc. (Laverde, 2010)

El arazá destaca como una de las especies nativas de la Amazonia de gran potencial, con perspectivas al desarrollo agroindustrial. La planta produce durante prácticamente el año entero y con esto, dependiendo del manejo de la plantación, se vislumbra la posibilidad de generar trabajo y renta continuamente en el campo, sin el problema de estacionalidad, común en diversos cultivos.

Gracias a todos los beneficios que nos brinda este fruto para mejorar el porcentaje nutricional de las personas, se tiene la convicción de que es importante dar a conocer y promover su consumo en la dieta diaria.

\section{OBJETIVO GENERAL}

Identificar y dar a conocer las propiedades nutricionales que aporta el yogurt de arazá, de tal manera que ayude a combatir problemas como el sobrepeso y la obesidad.

\section{OBJETIVOS ESPECÍFICOS}

- Desarrollar una investigación profunda y exhaustiva acerca de los beneficios que posee el arazá.

- Identificar los lugares potenciales donde se podría comercializar el yogurt de arazá en la ciudad de Guayaquil. 


\section{Análisis de Emprendimiento de Yogurt a base de Arazá en la Ciudad de Guayaquil}

Vol. 2, núm. Esp., (2018)

Eva María de Lourdes Loaiza Massuh; Holger Renato Ponce Arreaga; Hypathia Verónica Fiallos Veintimilla

- Incentivar el consumo de yogurt a base de arazá como una fuente alimenticia, aprovechando sus propiedades nutricionales.

\section{MARCO REFERENCIAL}

\section{BASES TEÓRICAS}

Ecuador en la última década ha venido atravesando por diferentes procesos estructurales, entre ellos la aparición de una cultura de emprendimiento e innovación. También resulta necesario conocer que es lo que motiva a los seres humanos a participar en el desarrollo de empresas, pequeños negocios, que impulsan el desarrollo económico de la ciudad de Guayaquil, y las variables que intervienen en los emprendimientos innovadores.

El proceso de cambio "ha venido acompañado de una serie de estrategias que acrecientan el interés y que despiertan el espíritu emprendedor de los ecuatorianos, impulsándolos a desarrollar nuevas propuestas de valor, destacándose las principales estrategias: cambio de matriz productiva, apertura de nuevos mercados y la consolidación de mercados externos". (Lideres, 2013).

En el estudio realizado por (Global Entrepreneurship Monitor GME, 2017), para impulsar el emprendimiento en el país entre otras cosas se necesita lo siguiente:

a) Mejorar las condiciones de entorno y financiamiento

b) Generar espacios de innovación y desarrollo de mercados 


\section{Análisis de Emprendimiento de Yogurt a base de Arazá en la Ciudad de \\ Guayaquil}

Vol. 2, núm. Esp., (2018)

Eva María de Lourdes Loaiza Massuh; Holger Renato Ponce Arreaga; Hypathia Verónica Fiallos

Veintimilla

c) Promover la vocación y competencias emprendedoras y propiciar una mayor conexión con el mundo académico y laboral

d) Promover redes de emprendedores; y,

e) Fomentar el uso de nuevas tecnologías de la información

Las funciones que cumplen los emprendedores y empresarios en una determinada sociedad constituyen un aspecto relevante del desempeño económico, al ser estos agentes del cambio social. Frecuentemente están asociados con el establecimiento de empresas pequeñas y medianas, con la asunción de capitales de riesgo y con la introducción de innovaciones. (Cobarrubias, 1696)

"La empresa es una entidad conformada básicamente por personas, aspiraciones, realizaciones, bienes materiales y capacidades técnicas y financieras; todo lo cual, le permite dedicarse a la producción y transformación de productos y/o la prestación de servicios para satisfacer necesidades y deseos existentes en la sociedad, con la finalidad de obtener una utilidad o beneficio". (Chirinos, 2011, pág. 7)

Además, el plan de negocios es un instrumento sobre el que se apoya un proceso de planificación sistemático y eficaz, con toda la información necesaria para evaluar un negocio y los lineamientos generales para ponerlo en marcha (Leuona, 2012)

Según (Drucker, La innovación y el empresario, 1986) expresa en su libro La innovación y el empresario innovador: El empresario innovador ve el cambio como una norma saludable. No 


\section{Análisis de Emprendimiento de Yogurt a base de Arazá en la Ciudad de Guayaquil}

Vol. 2, núm. Esp., (2018)

Eva María de Lourdes Loaiza Massuh; Holger Renato Ponce Arreaga; Hypathia Verónica Fiallos Veintimilla

necesariamente lleva a cabo el cambio él mismo, pero busca el cambio, responde a él y lo explota como una oportunidad, debido a esto es importante buscar nuevas formas de satisfacer las necesidades del mercado.

El yogurt es originario de los países del Medio Oriente, estos pueblos solían trasportar la leche fresca que se obtenía de los animales en grandes sacos de cuero. El calor y el contacto de la leche con el saco, permitía la reproducción de las bacterias acidas, las mismas que fermentaban la leche. El yogurt se transformó en un alimento básico desde la antigüedad. (A. Tamime, 2002)

Después de analizar la relevancia que tiene una mente misionaria y las empresas en la economía de un país, se puede decir que el lanzamiento de un producto innovador como es el yogurt de arazá tendrá viabilidad en el mercado de la ciudad de Guayaquil; el yogurt es un producto acidificado y coagulado que se obtiene a partir de la fermentación de la lactosa (azúcar de la leche) en ácido láctico por acción de microorganismos. El yogurt es la más conocida de las leches fermentadas y la de mayor consumo a nivel mundial. (EARLY, 2001)

\section{RECOLECCIÓN DATOS}

De acuerdo con las investigaciones de mercado realizadas en la Ciudad de Guayaquil, el 60\% de los encuestados no se preocupan por su salud y por ende la visión de esta a 20 años indica mucha incertidumbre. La mayor parte de los encuestados son consumidores reactivos, o sea personas que consumen productos para su salud por enfermedades específicas más no por prevención. Los hombres en su gran mayoría más consumidores reactivos que las mujeres. 


\section{Análisis de Emprendimiento de Yogurt a base de Arazá en la Ciudad de \\ Guayaquil}

Vol. 2, núm. Esp., (2018)

Eva María de Lourdes Loaiza Massuh; Holger Renato Ponce Arreaga; Hypathia Verónica Fiallos

Veintimilla

Esto resume que la tendencia en la actualidad, en cuanto al cuidado personal ha despuntado, por lo tanto, se considera importante mantener un buen estado físico exterior e interior. Sin embargo, existen personas que consumen productos cuando ya están enfermas, lo cual haría que ARAGURT sea más consumido por su exquisito sabor más que por su composición nutricional. Incluso en las encuestas sobre las razones de consumo de yogurt, el $45 \%$ de los encuestados lo comprarían por su sabor.

Las personas consumen un producto principalmente por su sabor, pero sin descartar los beneficios que este les pueda brindar a la salud, por lo que consideran que los beneficios se deben detallar en un cuadro nutricional impresos en afiches, trípticos, volantes y páginas Web.

\section{ANÁLISIS FODA}

Se presentan las "Fortalezas", "Oportunidades", "Debilidades" y "Amenazas" del proyecto, con el fin de que se trate de aprovechar y potenciar las fortalezas y las oportunidades, al mismo tiempo que trate de corregir o reducir los riesgos que conllevan las debilidades y amenazas.

\section{FORTALEZAS}

- Por su delicioso sabor, olor y aroma será fácil llegar a posibles consumidores e introducirlo en el mercado

- Debido a su gran porcentaje de Vitamina C y un elevado índice de Potasio será de gran aceptación en el mercado meta.

- Contribuir con el desarrollo socio-económico de las personas que se dedican al cultivo de esta fruta. 


\title{
Análisis de Emprendimiento de Yogurt a base de Arazá en la Ciudad de Guayaquil
}

Vol. 2, núm. Esp., (2018)

Eva María de Lourdes Loaiza Massuh; Holger Renato Ponce Arreaga; Hypathia Verónica Fiallos Veintimilla

\section{OPORTUNIDADES}

- Alianzas estratégicas con tiendas y amplias cadenas de supermercados en la ciudad de Guayaquil.

- Demanda creciente de alimentos saludables y funcionales basado en el arazá.

- La posibilidad de crecimiento de la empresa gracias a la amplia variedad de productos que se pueden derivar de esta fruta.

- Tener la posibilidad de ampliar el mercado a nivel nacional y exportar la pulpa hacia el extranjero.

\section{DEBILIDADES}

- Falta de conocimiento de la fruta y de sus beneficios por parte de la población a estudiar.

- Limitación de expandir la producción debido al poco cultivo de dicha fruta.

\author{
AMENAZAS \\ - Inestabilidad política, económica y social por la que atraviesa nuestro país. \\ - Una baja cosecha de esta fruta en gran parte del año. \\ - La gran variedad de productos sustitutos a los que se enfrenta.
}

\section{DESCRIPCIÓN PRODUCTO}

La empresa estará dedicada a la Producción y Comercialización del producto agroindustrial a base de materia prima y fruta. El producto ofrece un alimento lácteo que se obtiene mediante la 


\section{Análisis de Emprendimiento de Yogurt a base de Arazá en la Ciudad de \\ Guayaquil}

Vol. 2, núm. Esp., (2018)

Eva María de Lourdes Loaiza Massuh; Holger Renato Ponce Arreaga; Hypathia Verónica Fiallos

Veintimilla

fermentación bacteriana de la leche. Su textura y sabor tan particular le viene dado por la conversión de la lactosa (azúcar de la leche) en ácido láctico.

El propósito del mismo es brindar al público un yogurt dietético con la finalidad de cubrir las necesidades de los consumidores que padecen diversas enfermedades debido a un desorden alimenticio y a la rutina diaria.

\section{DESCRIPCIÓN Y CARACTERÍSTICAS FÍSICAS}

La fruta de arazá, es de color verde en estado inmaduro y ligeramente amarillento a amarillo dorado cuando alcanza la madurez, aproximadamente a los 90 días de la floración, Es exquisita, muy aromática, posee un exocarpo liso o aterciopelado, un mesocarpio carnoso de color amarillo de sabor fuertemente acido, de $10 \mathrm{~cm}$. de diámetro, con pesos comprendidos entre 200 gramos y puede llegar hasta 600 gramos y generalmente posee de 8 a 10 semillas.

La producción del arazá comienza al segundo año. La planta tiene simultáneamente flores y frutos, aunque existen períodos de cosecha como son los meses de noviembre a enero, abril a junio, y de septiembre a octubre. Se cultiva principalmente en la costa ecuatoriana y en pequeña escala en el Oriente, principalmente en la provincia de Pichincha (Noroccidente y Sto. Domingo), Los Ríos y en las provincias de Sucumbíos y Orellana.

La fruta es muy susceptible a sufrir daños por la manipulación y transporte, especialmente cuando está madura, por lo que la cosecha se debe realizar cuando el fruto está casi verde (pintón), aproximadamente a los 82 días después de la floración. 


\section{Análisis de Emprendimiento de Yogurt a base de Arazá en la Ciudad de Guayaquil}

Vol. 2, núm. Esp., (2018)

Eva María de Lourdes Loaiza Massuh; Holger Renato Ponce Arreaga; Hypathia Verónica Fiallos Veintimilla

La Guayaba Arazá es considerada como un alimento funcional, esto quiere decir que además de su valor nutricional, esta tiene una función especial en el organismo, en el caso del arazá esta previene y controla algunas enfermedades cardiovasculares (diabetes) y degenerativas como el cáncer, esto debido a su alto tenor de sustancias antioxidantes. Además, es una excelente fuente de vitamina $\mathrm{C}$ lo que contribuye a mantener un saludable sistema inmunológico, su aporte sustancial de pectina contribuye a ralentizar la absorción intestinal de azucares simples por tal motivo es recomendado en personas diabéticas pues al disminuir la velocidad de paso de los azucares del estómago al duodeno, se evita que aumenten de forma brusca los azucares en la sangre.

La presencia de pectinas también tiene un efecto beneficioso sobre los niveles de colesterol en la sangre.

Por ser una fruta rica en vitaminas, minerales y por tener una óptima combinación de

proteínas y carbohidratos puede ser tomado como SUPLEMENTO NUTRICIONAL preferiblemente en leche para maximizar sus propiedades. 


\section{Análisis de Emprendimiento de Yogurt a base de Arazá en la Ciudad de \\ Guayaquil}

Vol. 2, núm. Esp., (2018)

Eva María de Lourdes Loaiza Massuh; Holger Renato Ponce Arreaga; Hypathia Verónica Fiallos

Veintimilla

\section{Tabla 1: Contenido Nutricional por 100 gramos de pulpa de arazá:}

\begin{tabular}{|c|c|}
\hline \multicolumn{2}{|c|}{ Contenido nutricional arazá ( $100 \mathrm{~g}$ de pulpa) } \\
\hline Humedad $(\mathrm{g} / 100 \mathrm{~g})$ & 93.52 \\
\hline Extracto etéreo $(\mathrm{g} / 100 \mathrm{~g})$ & 1.43 \\
\hline Proteína $(\mathrm{g} / 100 \mathrm{~g})$ & 0.66 \\
\hline Fibra dietética (fracción sol.) (g/100g) & 0.61 \\
\hline Fibra dietética (fracción insol.) $(\mathrm{g} / 100 \mathrm{~g}$ ) & 2.85 \\
\hline Cenizas $(\mathrm{g} / 100 \mathrm{~g})$ & 0.13 \\
\hline Carbohidratos totales $(\mathrm{g} / 100 \mathrm{~g})$ & 4.26 \\
\hline Calorías/100g & 30 \\
\hline Calcio (mg/lit) & 0.136 \\
\hline Fósforo $(\mathrm{mg} / 100 \mathrm{~g})$ & 0.06 \\
\hline Hierro $(\mathrm{mg} / \mathrm{lt})$ & 0.841 \\
\hline Pro Vitamina A (B Carotenos) (mg/100g) & $0.03 \pm 0.0$ \\
\hline Vitamina B1 $(\mathrm{mg} / 100 \mathrm{~g})$ & $0.007 \pm 0.0$ \\
\hline Vitamina B2 $(\mathrm{mg} / 100 \mathrm{~g})$ & LND \\
\hline Vitamina C $(\mathrm{mg} / 100 \mathrm{~g})$ & $62.8 \pm 0.71$ \\
\hline Azúcares totales aproxim.(mg/100g) & 481 \\
\hline Glucosa (mg/100g) & $163.4 \pm 7.6$ \\
\hline Fructosa $(\mathrm{mg} / 100 \mathrm{~g})$ & $214 \pm 0.18$ \\
\hline Sacarosa $(\mathrm{mg} / 100 \mathrm{~g})$ & $104.2 \pm 2.1$ \\
\hline $\mathrm{pH}$ & 2.57 \\
\hline Acidez titulable $(\mathrm{g} / 100 \mathrm{~g})$ & 2.88 \\
\hline
\end{tabular}

\section{PRODUCCIÓN DEL ARAZÁ.}

En la actualidad existe mayor conciencia con respecto a la salud y la estética. Las personas desean comer bien pero también comer sano; es decir, con altos ingredientes nutritivos. Es de conocimiento de todos que por el ritmo de vida acelerado y los cambios de distintos factores producen efectos negativos en nuestra salud como los problemas cotidianos e presión arterial diabetes, que prohíben a las personas del consumo de determinados productos produciendo insatisfacción, en algunos casos enojo, cayendo en la resignación.

Para aspectos de agro-industrialización a pequeña escala la extracción de la pulpa de arazá es relativamente fácil. La pulpa constituye el $70 \%$ del peso del fruto fresco y tiene un 


\section{Análisis de Emprendimiento de Yogurt a base de Arazá en la Ciudad de Guayaquil}

Vol. 2, núm. Esp., (2018)

Eva María de Lourdes Loaiza Massuh; Holger Renato Ponce Arreaga; Hypathia Verónica Fiallos Veintimilla

rendimiento de 51 a 55\% de pulpa refinada. Una vez extraída la pulpa se puede guardar en bolsas o recipientes plásticos a menos de $10^{\circ} \mathrm{C}$.

La fruta se emplea en la preparación de jugos, néctar, helados y mermeladas. Dado el alto porcentaje de pulpa $(70 \%)$ se puede utilizar para combinar con otros frutales. La fruta también tiene potencial para la extracción de los principios aromáticos por su olor muy agradable y exótico que podrá ser utilizada en la industria de perfumes.

\section{DATOS DEMOGRÁFICOS}

El consumo de yogurt tiene un arraigo muy fuerte en ciudades europeas como en Holanda que el consumo es de 38 kilogramos por persona, no sucede lo mismo en América, mientras en Estados Unidos el consumo es apenas por debajo de los tres kilogramos per cápita, en México la cifra es de ocho kilogramos por persona, lo que es el equivalente a una cuchara sopera al día.

\section{IMPACTO ECONÓMICO Y SOCIAL DEL PROYECTO.}

La idea de constituir una microempresa surge del deseo de participar activamente en el desarrollo de la economía, en mejorar la calidad de vida y las condiciones sociales de las personas de la ciudad de Guayaquil, lo cual se logra a partir del diseño e innovación de los productos que se ofrecen a los consumidores, la relación de negocios con los proveedores, la creación de oportunidades de empleo y la generación de utilidades para los socios. 


\section{Análisis de Emprendimiento de Yogurt a base de Arazá en la Ciudad de}

Guayaquil

Vol. 2, núm. Esp., (2018)

Eva María de Lourdes Loaiza Massuh; Holger Renato Ponce Arreaga; Hypathia Verónica Fiallos

Veintimilla

\section{OFERTA}

Con respecto al arazá, aun no existe una oferta de este producto establecida a nivel nacional. Sin embargo, es preciso mencionar el principal país exportador del arazá como tal para tener una idea más clara de cuanto es la cantidad a nivel mundial de las exportaciones del arazá.

En la siguiente gráfica se demuestra a los principales países exportadores del arazá.

Tabla 2: Principales productores del arazá

\begin{tabular}{|c|c|}
\hline Exportadores & $\begin{array}{l}\text { Valor de producción en } \\
\text { TM }\end{array}$ \\
\hline Brasil & $664.286,00$ \\
\hline Colombia & $474.442,00$ \\
\hline Perú & $6.000,00$ \\
\hline Costa Rica & $356.320,00$ \\
\hline Total & $1.501 .048,00$ \\
\hline
\end{tabular}

Fuente: FAO (2010)

Ecuador cuenta con 8.032.517 de hectáreas con algún tipo de uso agropecuario, en las que se ubican 842,882 Unidades de Producción Agrícola, UPAS. Aproximadamente el 75\% de la propiedad agrícola se concentra en manos de productores que poseen menos de 10 hectáreas.

No existen estadísticas oficiales sobre pequeños productores agropecuarios, la dificultad de relacionar a la pequeña producción campesina únicamente por el tamaño del predio, conduce 


\section{Análisis de Emprendimiento de Yogurt a base de Arazá en la Ciudad de Guayaquil}

Vol. 2, núm. Esp., (2018)

Eva María de Lourdes Loaiza Massuh; Holger Renato Ponce Arreaga; Hypathia Verónica Fiallos Veintimilla

a identificar otras variables, como la importancia relativa en la conformación del ingreso familiar de la utilización de los recursos de la finca.

Las organizaciones de comercio justo, por ejemplo, definen como pequeños productores a aquellos que no dependen estructuralmente de trabajo contratado permanente, administrando su plantación principalmente con su propio trabajo y el de su familia. 8

En la región amazónica ecuatoriana, una de las frutas prioritarias identificadas para este estudio debido a su potencialidad y oportunidad de mercado es el Arazá. Stipitata, E. (2000)

En Valle del Cauca, Colombia, ha cultivado 20 hectáreas de arazá para el fortalecimiento competitivo en el mercado nacional con miras a la exportación de la pulpa como tal o como subproductos: mermeladas, néctares, licor, salsas agridulces, y otros.

Existen asociaciones de campesinos con metas de crecimiento para poder competir internacionalmente

La producción de arazá en Colombia se ha concentrado en las regiones cálidas y húmedas del país, especialmente en el sur, donde los principales productores están en los departamentos de Guavira, Caquetá y Putumayo, Caquetá 200 hectáreas sembradas, que equivale 840 toneladas al año. 


\section{Análisis de Emprendimiento de Yogurt a base de Arazá en la Ciudad de \\ Guayaquil}

Vol. 2, núm. Esp., (2018)

Eva María de Lourdes Loaiza Massuh; Holger Renato Ponce Arreaga; Hypathia Verónica Fiallos

Veintimilla

\section{DEMANDA}

La demanda de frutas exóticas se ha incrementado en los últimos años. La búsqueda de nuevos sabores para satisfacer finos y exquisitos paladares ha hecho que frutas como el arazá sean utilizadas para la elaboración de postres, jugos, mermeladas, licores, etc.

Mercados internacionales, como Estados Unidos, Ucrania, España, Japón, Francia, Alemania, Holanda, Bélgica son grandes consumidores de frutas exóticas.

En los últimos 8 años el Gobierno ha buscado darle un cambio a la matriz productiva del Ecuador, con apoyo de las instituciones como la de PROECUADOR, MAGAP, entre otras.

En la constate búsqueda de identificar mercados potenciales, PROECUADOR, se ha preocupado por realizar ferias internaciones de frutas y verduras y así poder posicionar el producto ecuatoriano en mercados internacionales.

Sin embargo, en Ecuador, dicha fruta no se encuentra relacionada a la alimentación diaria de la población; por lo cual se ha buscado la alternativa de exportar dicho producto a países con gran demanda, es por ello que la creación de un producto que dinamice el gusto de lo población ecuatoriana podría poseer gran acogida y aceptación del producto.

\section{COMERCIALIZACIÓN}

CANALES DE DISTRIBUCIÓN 


\section{Análisis de Emprendimiento de Yogurt a base de Arazá en la Ciudad de Guayaquil}

Vol. 2, núm. Esp., (2018)

Eva María de Lourdes Loaiza Massuh; Holger Renato Ponce Arreaga; Hypathia Verónica Fiallos Veintimilla

Se utilizará el Canal de Distribución Nivel 1, que trata el medio IMDUSTRIA - PUNTOS DE VENTA - CONSUMIDOR, que nos brinda la ventaja de ahorrar gastos adicionales de venta e inversión en puntos de venta, lo cual no convendría debido a que solo contamos con un solo producto por el momento. Una desventaja sería ajustarnos a políticas por parte de los puntos de venta, ellos tendrían el poder de negociación.

\section{PUNTOS DE VENTA}

Cadena de Grandes supermercados como Supermaxi, Megamaxi, Mi comisariato, Tía, Gran AKÍ, Mini Mercado, ubicados en diferentes sectores de la urbe.

\section{PROPUESTA DEL PROYECTO}

Este proyecto pretende la realización de una microempresa que se dedique a la elaboración y comercialización de productos naturales a base de la fruta del arazá para el beneficio de las personas que habitan en la ciudad de Guayaquil. 


\section{Análisis de Emprendimiento de Yogurt a base de Arazá en la Ciudad de \\ Guayaquil}

Vol. 2, núm. Esp., (2018)

Eva María de Lourdes Loaiza Massuh; Holger Renato Ponce Arreaga; Hypathia Verónica Fiallos

Veintimilla

Tabla 3 Cuadros de datos para calcular el Punto de Equilibrio del yogurt de arazá.

\begin{tabular}{|c|c|c|}
\hline \multicolumn{3}{|c|}{ PUNTO DE EQUILIBRIO (YOGURT DE ARAZÁ) } \\
\hline Precio de Venta Unitario & & $\$ 1,30$ \\
\hline Unidades Vendidas & & 3000 \\
\hline Ingreso Total & & $\$ 3.907,89$ \\
\hline Costo Fijo Total & & $\$ 2.215,00$ \\
\hline Costo Variable Total & & $\$ 1.260,00$ \\
\hline Costo Variable Unitario $(\mathrm{Cvu})=$ & CVT / $\mathrm{N}^{\circ}$ unid. Vendidas & $\$ 0,42$ \\
\hline \multicolumn{2}{|c|}{ Cantidad de Equilibrio=CFT/(P-Cvu) } & 2510 \\
\hline Precio de Equilibrio $=$ & & $\$ 3.269,01$ \\
\hline
\end{tabular}

Tabla 4: Cuadro de valores obtenidos en el Punto de Equilibrio.

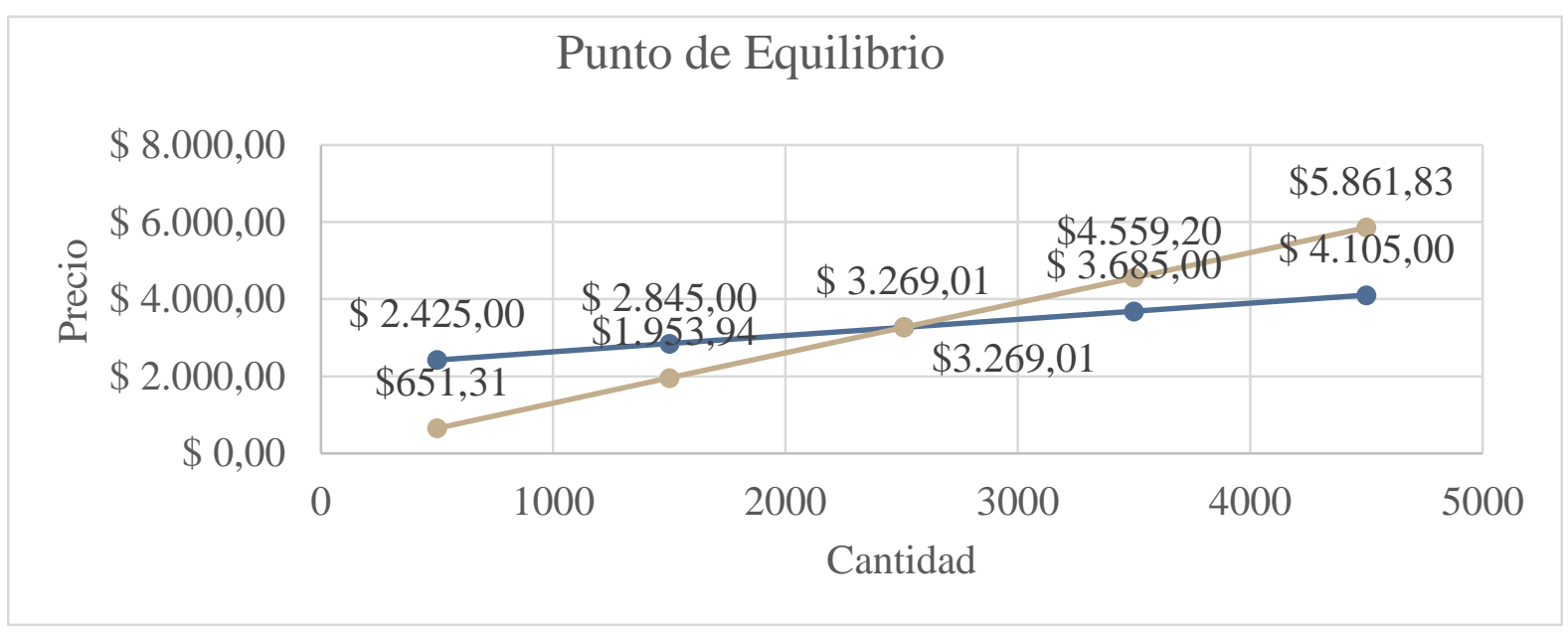




\section{Análisis de Emprendimiento de Yogurt a base de Arazá en la Ciudad de Guayaquil}

Vol. 2, núm. Esp., (2018)

Eva María de Lourdes Loaiza Massuh; Holger Renato Ponce Arreaga; Hypathia Verónica Fiallos Veintimilla

\begin{tabular}{|l|l|l|l|l|l|l|}
$\begin{array}{l}\text { Precio Vta. } \\
\text { Unit. }\end{array}$ & Cantidad & $\begin{array}{l}\text { Ingreso } \\
\text { Total }\end{array}$ & $\begin{array}{l}\text { Costos } \\
\text { Fijos }\end{array}$ & $\begin{array}{l}\text { Costo Var. } \\
\text { Unit. }\end{array}$ & $\begin{array}{l}\text { Costo Var. } \\
\text { Tot. }\end{array}$ & $\begin{array}{l}\text { Costo } \\
\text { Total }\end{array}$ \\
\hline$\$ 1,30$ & 500 & $\$ 651,31$ & $\$ 2.215,00$ & $\$ 0,42$ & $\$ 210,00$ & $\$ 2.425,00$ \\
\hline$\$ 1,30$ & 1500 & $\$ 1.953,94$ & $\$ 2.215,00$ & $\$ 0,42$ & $\$ 630,00$ & $\$ 2.845,00$ \\
\hline$\$ 1,30$ & 2510 & $\$ 3.269,01$ & $\$ 2.215,00$ & $\$ 0,42$ & $\$ 1.054,01$ & $\$ 3.269,01$ \\
\hline$\$ 1,30$ & 3500 & $\$ 4.559,20$ & $\$ 2.215,00$ & $\$ 0,42$ & $\$ 1.470,00$ & $\$ 3.685,00$ \\
\hline$\$ 1,30$ & 4500 & $\$ 5.861,83$ & $\$ 2.215,00$ & $\$ 0,42$ & $\$ 1.890,00$ & $\$ 4.105,00$ \\
\hline
\end{tabular}

\section{PRECIO}

Como el gramo de yogurt cuesta $0.42 \mathrm{ctvs}$; se lo ha convertido en kilogramos dando un precio de $\$ 1.30$

\section{ESTUDIO TÉCNICO}

\section{TAMAÑO}

\section{MERCADO}

Como se lo especificó en los datos demográficos y las características de los consumidores, el mercado objetivo de este proyecto será Guayaquil debido a que el consumo de yogurt no posee un arraigo muy fuerte en esta ciudad, sin embargo, las personas que habitan en esta ciudad están abierto al consumo de yogurt por lo tanto sería un mercado donde se podría implementar este proyecto. 


\section{Análisis de Emprendimiento de Yogurt a base de Arazá en la Ciudad de}

Guayaquil

Vol. 2, núm. Esp., (2018)

Eva María de Lourdes Loaiza Massuh; Holger Renato Ponce Arreaga; Hypathia Verónica Fiallos

Veintimilla

\section{DISPONIBILIDAD DE LA MATERIA PRIMA}

Esta fruta se cultiva en mayores extensiones en la costa ecuatoriana, especialmente en el cantón La Concordia, aunque pequeñas plantaciones se formaron, con anterioridad (1982), en la Amazonía, principalmente en Napo, Orellana, Sucumbíos y Pastaza.

La producción se inicia al tercer año para estabilizarse el quinto, con rendimientos que pueden alcanzar hasta las 14 toneladas por hectárea al año, dependiendo de la fertilización, abonamientos, cuidados fitosanitarios y manejo general. El período de producción puede alcanzar los 25 años. La recolección debe ser inmediata para no dejar madurar mucho la fruta.

Según el III Censo Nacional Agropecuario efectuado en el año 2002, se encuentra registrada oficialmente 579 hectáreas en la provincia de Esmeraldas.

El arazá es cultivado en toda la región amazónica del Ecuador. La podemos encontrar en la zona norte como en los cantones de Cascales, Lago Agrio, pertenecientes a la provincia de Sucumbíos. Shushufindi, Sacha, Coca y Loreto cantones que pertenecen a la provincia de Orellana. En la zona centro tenemos a los cantones Archidona y Tena que pertenecen a la provincia de Napo. También los cantones de Mera y Puyo pertenecientes a la provincia de Pastaza. Los cantones de Palora y Sucúa de la provincia de Morona Santiago. Y en la zona sur encontramos cultivos en los cantones de Yantzaza, El Pangui, Nangaritza y Centinela del Cóndor que pertenecen a la provincia de Zamora Chinchipe. 


\section{Análisis de Emprendimiento de Yogurt a base de Arazá en la Ciudad de Guayaquil}

Vol. 2, núm. Esp., (2018)

Eva María de Lourdes Loaiza Massuh; Holger Renato Ponce Arreaga; Hypathia Verónica Fiallos Veintimilla

En provincias de la costa como Manabí y Guayas también se puede encontrar cultivos de Arazá..En las provincias de Pichincha y Santo Domingo de los Tsáchilas también encontramos Arazá con un aproximado de 300 hectáreas cultivadas.

A continuación, un cuadro con la superficie sembrada y su producción del cultivo de arazá.

Tabla 5: Superficie Sembrada y construcción del arazá

\begin{tabular}{|l|c|c|}
\hline Provincias & Superficie Sembrada & Produccion anual TM \\
\hline Morona Santiago & 26,00 & 2,24 \\
\hline Napo & 10,00 & 0,86 \\
\hline Orellana & 20,00 & 1,73 \\
\hline Pastaza & 18,00 & 1,55 \\
\hline Sucumbios & 20,00 & 1,73 \\
\hline Prichincha & 75,00 & 6,47 \\
\hline Santo domingo de los Tsachilas & 190,00 & 16,40 \\
\hline Esmeralda & 579,00 & 49,97 \\
\hline Total & 938,00 & 80,95 \\
\hline
\end{tabular}

Fuente: INEC 2013

La fruticultura es una actividad agrícola de limitada en la Amazonia Brasilera, principalmente de las especies frutícolas nativas.

Tradicionalmente, la mayor parte de las brutas que llegan a los mercados y ferias provienen de la extracción de árboles silvestres y de pequeños huertos familiares. Brasil es el país de Suramérica con mayor producción de frutas tropicales amazónicas (44\%), seguido de Colombia, Ecuador, Perú y de último Bolivia. 


\section{Análisis de Emprendimiento de Yogurt a base de Arazá en la Ciudad de}

Guayaquil

Vol. 2, núm. Esp., (2018)

Eva María de Lourdes Loaiza Massuh; Holger Renato Ponce Arreaga; Hypathia Verónica Fiallos

Veintimilla

\section{DISPONIBILIDAD DE MANO DE OBRA}

En Ecuador, la disponibilidad de la mano de obra si es alta, básicamente en lo que se incurría es en capacitaciones al personal para que aprenda como elaborar el yogurt de arazá aplicando las Buenas Prácticas de Manufactura.

El salario de dicha mano de obra en el Ecuador es de $\$ 354,00$. Sin embargo, hay que considerar que para elaborar una $5 \mathrm{Kg}$. Del yogurt de arazá la utilización de la mano de obra es de apenas de entre 1-2 horas/hombre. Por lo que no es necesario incorporar tanta mano de obra en nuestro proceso productivo.

\section{CAPACIDAD INSTALADA}

La capacidad instalada en nuestro proyecto será del 100\% para tratar que los costos se mantengan bajos. Es decir, que se tratará de no tener una capacidad instalada ociosa para no tener altos costos y así tratar de no tener altos precios para que nuestra empresa tenga alta competitividad.

\section{LOCALIZACIÓN}

\section{MACRO-LOCALIZACIÓN}

Dentro del mejor sitio para ubicar nuestra planta de procesamiento del yogurt de arazá, es necesario tener en consideración que la localización óptima será aquella que permita obtener la 


\section{Análisis de Emprendimiento de Yogurt a base de Arazá en la Ciudad de Guayaquil}

Vol. 2, núm. Esp., (2018)

Eva María de Lourdes Loaiza Massuh; Holger Renato Ponce Arreaga; Hypathia Verónica Fiallos Veintimilla

máxima producción maximizando los beneficios y reduciendo al mínimo posible los costes de producción, en este caso de transportación.

\section{- Lugar / Materia prima}

Con respecto a la localización, será más factible tener nuestra empresa de producción cercana a la materia prima, sin embargo, debido al nivel de comercialización en la ciudad de Guayaquil se determina que se empleará en dicha ciudad.

\section{- Abastecimiento de los servicios}

Como es de saber, entre la provincia del Guayas cuenta con todos los servicios que se requieren para, especialmente, el proceso productivo. Tal es el caso de las inversiones que se han hecho en alcantarillado, agua potable, luz y teléfono.

\section{- Factibilidad dentro del área establecida.}

La factibilidad de invertir en una pequeña parcela para llevar a cabo el proceso productivo del yogurt de arazá. Debido a la cercanía que existe entre la materia prima y la planta de procesamiento.

\section{MICRO-LOCALIZACIÓN}

\section{- Infraestructura socioeconómica}

Dentro de este aspecto se toma en cuenta la disponibilidad de transporte, mano de obra clima y servicios. 


\section{Análisis de Emprendimiento de Yogurt a base de Arazá en la Ciudad de Guayaquil}

Vol. 2, núm. Esp., (2018)

Eva María de Lourdes Loaiza Massuh; Holger Renato Ponce Arreaga; Hypathia Verónica Fiallos

Veintimilla

Como ya es de saber, la ciudad de Guayaquil, posee gran acogida de productos, así como de nuevos emprendimientos por lo cual la implementación de un nuevo producto al mercado que sea nutritivo y saludable para el consumidor logre ser viable para la empresa; sin embargo, la movilidad del transporte de la materia prima sería un factor de gran importancia, por ello el personal capacitado y los medios adecuados será necesario para la adquisición de dichos servicios y la producción del yogurt de arazá.

\section{- Infraestructura de servicios}

Los servicios necesarios en el proceso productivo del yogurt de arazá son básicamente luz, agua y alcantarillado. Los mismos que si están disponibles en el sitio tomado para llevar a cabo la planta de procesamiento para la elaboración del yogurt de arazá.

Cabe recalcar la importancia que el gobierno nacional ha realizado su inversión en vías de acceso (carreteras) lo cual ha sido y es beneficioso para toda actividad productivo que se lleve a cabo. La energía eléctrica con las grandes hidroeléctricas que se han realizado que el proceso productivo sea de manera eficiente y eficaz. 


\section{Análisis de Emprendimiento de Yogurt a base de Arazá en la Ciudad de Guayaquil}

Vol. 2, núm. Esp., (2018)

Eva María de Lourdes Loaiza Massuh; Holger Renato Ponce Arreaga; Hypathia Verónica Fiallos Veintimilla

\section{ENCUESTAS DE VALORACIÓN DEL PRODUCTO}

¿Conoce usted la fruta llamada arazá?

\begin{tabular}{|l|l|l|}
\hline POBLACIÓN & SI & NO \\
\hline MUJER & 15 & 10 \\
\hline HOMBRE & 8 & 17 \\
\hline TOTAL & $\mathbf{2 3}$ & $\mathbf{2 7}$ \\
\hline
\end{tabular}

De las 50 personas que han sido encuestadas entre los hombres y mujeres nos da un resultado que el $46 \%$ conoce la fruta llamada arazá y el $64 \%$ restante no la conoce.

¿Ah escuchado usted alguna vez hablar del yogurt de arazá?

\begin{tabular}{|l|l|l|}
\hline POBLACIÓN & SI & NO \\
\hline MUJER & 4 & 20 \\
\hline HOMBRE & 2 & 22 \\
\hline TOTAL & $\mathbf{8}$ & $\mathbf{4 2}$ \\
\hline
\end{tabular}

De las 50 personas encuestadas $16 \%$ habían escuchado de este producto y el $84 \%$ no habían escuchado hablar del yogurt de arazá, aunque varias personas acotaron que habían escuchado hablar de otros productos derivados de esta fruta como mermeladas entre otros. 


\section{Análisis de Emprendimiento de Yogurt a base de Arazá en la Ciudad de}

Guayaquil

Vol. 2, núm. Esp., (2018)

Eva María de Lourdes Loaiza Massuh; Holger Renato Ponce Arreaga; Hypathia Verónica Fiallos

Veintimilla

\section{¿Compraría usted este producto? (yogurt de arazá)}

\begin{tabular}{|l|l|l|}
\cline { 2 - 3 } & SOBLACIÓN & NO \\
\hline MUJER & 15 & 5 \\
\hline HOMBRE & 25 & 5 \\
\hline TOTAL & $\mathbf{4 0}$ & $\mathbf{1 0}$ \\
\hline
\end{tabular}

De las 50 personas que han sido encuestadas entre los hombres y mujeres nos da un resultado que el $80 \%$ estaría dispuesto a comprar el yogurt de arazá y el $20 \%$ no.

¿Sabe usted que el yogurt de arazá es saludable y nutritivo?

\begin{tabular}{|l|l|l|}
\hline POBLACIÓN & SI & NO \\
\hline MUJER & 10 & 13 \\
\hline HOMBRE & 12 & 15 \\
\hline TOTAL & $\mathbf{2 2}$ & $\mathbf{2 8}$ \\
\hline
\end{tabular}

De las 50 personas que han sido encuestado entre los hombres y mujeres nos da un resultado que el $44 \%$ sabe que el arazá es saludable y nutritivo el $56 \%$ restante no lo sabía. 


\section{Análisis de Emprendimiento de Yogurt a base de Arazá en la Ciudad de Guayaquil}

Vol. 2, núm. Esp., (2018)

Eva María de Lourdes Loaiza Massuh; Holger Renato Ponce Arreaga; Hypathia Verónica Fiallos Veintimilla

¿Pagaría usted el valor de \$1,20 por la compra del yogurt de arazá?

\begin{tabular}{|l|l|l|}
\hline POBLACIÓN & SI & NO \\
\hline MUJER & 20 & 4 \\
\hline HOMBRE & 23 & 3 \\
\hline TOTAL & $\mathbf{4 3}$ & $\mathbf{7}$ \\
\hline
\end{tabular}

De las 50 personas que han sido encuestadas entre los hombres y mujeres nos da un resultado que el $86 \%$ estaría dispuesto a pagar el precio establecido, por el yogurt mientras que el $14 \%$ no lo pagaría.

\section{CONCLUSIONES}

- El yogurt de arazá es un producto con aceptación en el mercado, debido a su exquisito sabor y por los beneficios de salud que brinda.

- Es muy importante promover este yogurt, como un producto básico del consumo diario, dejando de lado lo que son las comidas chatarras que solo perjudican el bienestar de las personas

- Las investigaciones del mercado realizadas evidenciaron la favorable actitud de los consumidores potenciales hacia el uso del yogurt en su alimentación diaria. 


\section{Análisis de Emprendimiento de Yogurt a base de Arazá en la Ciudad de \\ Guayaquil}

Vol. 2, núm. Esp., (2018)

Eva María de Lourdes Loaiza Massuh; Holger Renato Ponce Arreaga; Hypathia Verónica Fiallos

Veintimilla

\section{RECOMENDACIONES}

- Establecer estrategias, procesos y procedimientos, los cuales deben ser utilizados efectivamente, para el logro de los objetivos propuestos.

- Es necesario introducir un producto con estas características en el mercado, no sólo por la rentabilidad que el negocio demuestra, sino también por las implicaciones positivas en la mejora de hábitos alimenticios y salud de la población.

- El gobierno debe promover la venta de alimentos saludables en los bares escolares.

\section{REFERENCIAS BIBLIOGRÁFICAS}

Ariza, C. A. (2001). Especies promisorias de la Amazonia: conservación, manejo y utilización del germoplasma. Bogotá. Salvador Rojas González Ed

Astudillo, M. et al, (2006). Proyecto de tesis para la elaboración y comercialización de Batiyogurt en Guayaquil. Recuperado de https://www.dspace.espol.edu.ec/bitstream/123456789/1218/1/2371.pdf

A. Tamime, R. R. (2002). Yogurt Salazonado. Nutrición, 55. Chirinos, M. (2011). Definición de Empresa. Ecuador.

Cobarrubias, I. (1696). Emprendedores y Empresarios: un enfoque Institucional. Contribuciones a la economía.

Drucker, P. (1986). La innovación y el empresario. En P. Drucker, La innovación y el empresario.

EARLY, R. (2001). Tecnología de los productos lácteos. En T. d. lácteos, Tecnología de los productos lácteos (pág. 40). Zaragoza - España: Acribia.

Martillo, I. (2014) RUTA AMAZÓNICA ARAZÁ. Revista caribeña de Ciencias Sociales. Recuperado de http://xn--caribea-9za.eumed.net/fruta-araza/ 


\section{Análisis de Emprendimiento de Yogurt a base de Arazá en la Ciudad de Guayaquil}

Vol. 2, núm. Esp., (2018)

Eva María de Lourdes Loaiza Massuh; Holger Renato Ponce Arreaga; Hypathia Verónica Fiallos Veintimilla

Laverde, J. (2010). Estudio de las condiciones óptimas para la obtención de jugo clarificado de arazá, mediante procesos enzimático y membranario. Quito: Ecuador. Escuela Politécnica Nacional.

Leuona, D. T. (2012). Apuntes de plan de negocios. Ecuador

Sánchez, G. (2, septiembre, 2005). La Hora. El arazá fruta de doble sabor, Recuperado de http://lahora.com.ec/index.php/noticias/show/1101420826/1/Cultivo_del_araz\%C3\%A1_en_nue stro_pa\%C3\%ADs.html\#.VtDIBX3Hdiv

Simbaña, J (2010). Estudio de factibilidad para la creación de una empresa dedicada a la producción y comercialización de yogurt de frutas amazónicas no tradicionales (arazá, borojó y guayaba) en la ciudad de Tena. Repositorio http://repositorio.ug.edu.ec/bitstream/redug/8334/1/TESIS\%20\%20ARAZA\%20GRACE\%20M ONTES.pdf

Stipitata, E. (2000). Cultivo y utilización. Recuperado de http://www.otca.info/portal/admin/_upload/publicacoes/SPT-TCA-VEN-SN-araza.pdf

Vargas, A., \& Rivera Camelo, Á., \& Narváez Cuenca, C. (2005). Capacidad antioxidante durante la maduración de arazá (Eugenia stipitata Mc Vaugh). Revista Colombiana de Química, 34 (1), 57-65. 